\title{
Cervical Spine Alignment in Helmeted Skiers and Snowboarders With Suspected Head and Neck Injuries: Comparison of Lateral C-spine Radiographs Before and After Helmet Removal and Implications for Ski Patrol Transport
}

\author{
Jared Murray, BA; David A. Rust, MD \\ From the University of Minnesota School of Medicine, Duluth Campus (Mr Murray) and St. Luke's Hospital, Orthopedics and Sports Medicine, \\ Whiteside Institute for Clinical Research and the Spirit Mountain Ski Partol, Duluth, MN (Dr Rust).
}

\begin{abstract}
Objective.-Current protocols for spine immobilization of the injured skier/snowboarder have not been scientifically validated. Observing changes in spine alignment during common rescue scenarios will help strengthen recommendations for rescue guidelines.

Methods. - Twenty-eight healthy volunteers (18 men, 10 women) age 47 \pm 17 (range 20-73) (mean \pm SD with range) underwent a mock rescue in which candidate patrollers completing an Outdoor Emergency Care course performed spine immobilization and back boarding in 3 scenarios: 1) Ski helmet on, no c-collar; 2) helmet on, with c-collar; and 3) helmet removed, with c-collar. After each scenario, a lateral radiograph was taken of the cervical spine to observe for changes in alignment.

Results.-Compared with the control group (helmet on, no collar), we observed 9 degrees of increased overall (occiput-C7) cervical extension in the helmet on, with collar group $(P<.001)$, and 17 degrees in the helmet off, with collar group $(P<.001)$. There was increased extension at the occiput-C2 intersegment in the helmet on, with collar group $(9$ degrees, $P<.001)$ and at both the occiput-C2 (9 degrees, $P<.001)$ and C2-C7 (8 degrees, $P<.001)$ intersegments in the helmet off, with collar group.

Conclusion.- -Ski helmet removal and c-collar application each leads to increased extension of the cervical spine. In the absence of other clinical factors, our recommendation is that helmets should be left in place and c-collars not routinely applied during ski patrol rescue.
\end{abstract}

Keywords: ski, snowboard, helmet, spine immobilization, c-collar, ski patrol

\section{Introduction}

Alpine winter sports are popular recreational activities with an estimated 19.8 million participants annually in the United States alone. ${ }^{1}$ According to the National Ski Area Association, there are 470 ski areas in the United States, with 57.1 million visits that generate $\$ 7.3$ billion annually in direct spending. ${ }^{2}$

Injuries are common among skiers and snowboarders with 1 study showing approximately 230 injuries per

Corresponding Author: David A. Rust, MD, St. Luke's Orthopedics and Sports Medicine, 1012 East 2nd St., Campus A Bldg Level 5, Duluth, MN 55805; e-mail: rustdave@ hotmail.com.

Submitted for publication October 2016.

Accepted for publication March 2017.
100,000 mountain visits. Head and neck injuries comprise more than $10 \%$ of these injuries and are more common in younger athletes and snowboarders. ${ }^{3}$ Spinal cord injuries are rare but potentially devastating. According to the National Spinal Cord Statistical Center there are approximately 17,000 new spinal cord injuries in the United States annually, of which about $9 \%$ result from sports and recreation. Winter sports and snow skiing have one of the highest frequencies of spinal cord injury. ${ }^{4}$ Severe injuries and fatalities among alpine winter athletes typically involve closed head or spinal injury. In 2011-12 there were 54 fatalities and 51 severe injuries (coma, paralysis) reported in 51 million skier visits by the National Ski Area Association. $^{2}$ 
The National Ski Patrol was started in 1930 by Charles "Minnie" Dole and is a national organization with more than 28,000 volunteers that provide first aid, triage, and emergency medical care and transportation to injured skiers and snowboarders. National Ski Patrol patrollers are trained as Outdoor Emergency Care (OEC) technicians having completed a course consisting of 80100 hours of classroom and practical skills training and testing as well as spending 1 year as a candidate patroller to become a certified patroller. Patrollers must also attend an annual "refresher" course covering approximately one third of the OEC curriculum to maintain certification. As patrollers, OEC technicians provide initial resuscitation, stabilization, triage, and transport, not invasive or advanced medical treatments, and care is usually dictated by both national and local procedures and protocols. ${ }^{5,6}$

As outlined in the OEC Manual, 5th edition, patrollers must have an understanding of head and spinal injury mechanisms, anatomy, physiology, and pathology as well as rescue skills including assessment of the neurologic injuries, manual spinal stabilization, sizing and applying a c-collar, placing patients on a long spine board, and helmet removal. ${ }^{7}$ Rescues may require toboggan, snowmobile, or even airlift in order to cross rugged and remote terrain. Thus, it is felt that athletes with suspected head/neck injuries have appropriate stabilization before transport.

Helmet use among alpine skiers and snowboarders has been shown to reduce the risk of closed head injury by up to $60 \%$ without an increased incidence of spinal trauma. ${ }^{8-13,23}$ The use of helmets among recreational skiers and snowboarders has increased steadily in the past decade from $25 \%$ in $2002-03$ to $67 \%$ in $2011-12$, and helmet use is now mandatory in the Canadian Province of Nova Scotia. ${ }^{14}$

Studies in other helmeted sports such as football and hockey have helped create guidelines for the emergency transportation of athletes with suspected head/neck injuries regarding the appropriate timing and location of helmet removal. ${ }^{15-18}$ To date there have been no studies that address this issue for alpine winter sports, and ski patrollers currently must rely solely on clinical judgement to decide when to remove a helmet rather than scientific evidence, leading to potential confusion and variation in practice.

The purpose of the current study was to observe changes in cervical spine alignment after the addition of a cervical collar to a helmeted skier, and after helmet removal and c-collar application in the mock-injured athlete stabilized on a spinal backboard by obtaining lateral c-spine radiographs in 3 common scenarios: 1) helmet on, without a cervical collar; 2) helmet on, with a cervical collar; and 3) helmet removed, with a cervical collar. We hypothesize that observing changes in spinal alignment in these scenarios will help establish evidence-based guidelines for the safe transport of alpine winter athletes with suspected head/neck injuries.

\section{Methods}

Approval to conduct research on human subjects was obtained from the Institutional Review Board at St Luke's Hospital and the Whiteside Research Institute, Duluth, MN. All volunteers were provided detailed information about the study and the risks of ionizing radiation and provided informed consent to participate. $^{19}$

Twenty-eight healthy adult volunteers (18 men, 10

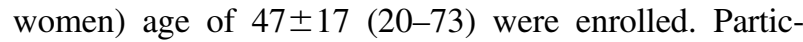
ipants were current and candidate ski patrollers who agreed to participate in a scenario-based exercise in which helmeted alpine athletes with suspected cranial/ cervical injuries were assessed and stabilized by ski patrol candidates completing an Outdoor Emergency Care course under the supervision of experienced course instructors. Volunteers were asked to bring their own personal ski wear (helmet and jacket) and were excluded from participation if they were age $<18$, known to be pregnant, or if they had any history of prior cervical spine injury or surgery.

Helmeted volunteers with mock head/neck injuries were stabilized on a long spine back board and had a lateral radiograph of the c-spine taken after each of 3 rescue scenarios. In scenario 1 (helmet on, no collar), the helmet was left in place and the patient immobilized directly on the spinal board without a c-collar using stable blocks to secure the head and helmet. For each individual, this scenario served as a control as it would be assumed to be the least time consuming and require the least spinal manipulation to perform. In scenario 2 (helmet on, with collar) the helmet was left in place and a cervical collar was applied to provide additional stabilization to the spine. In scenario 3 (helmet off, with collar) the helmet was removed and a cervical collar was applied using a standard 2 or 3 person rescue technique (Figure 1) taught in the OEC manual, and the length of time needed to perform the helmet removal and stabilization was recorded.

Radiographic analysis of the x-ray images was performed blindly by 2 independent observers and was identical to the technique used by Swenson et al in their study on American football players. ${ }^{15}$ Observers used digital imaging software to mark the posterior occiput line, posterior dens line, and C7 inferior end plate line on 


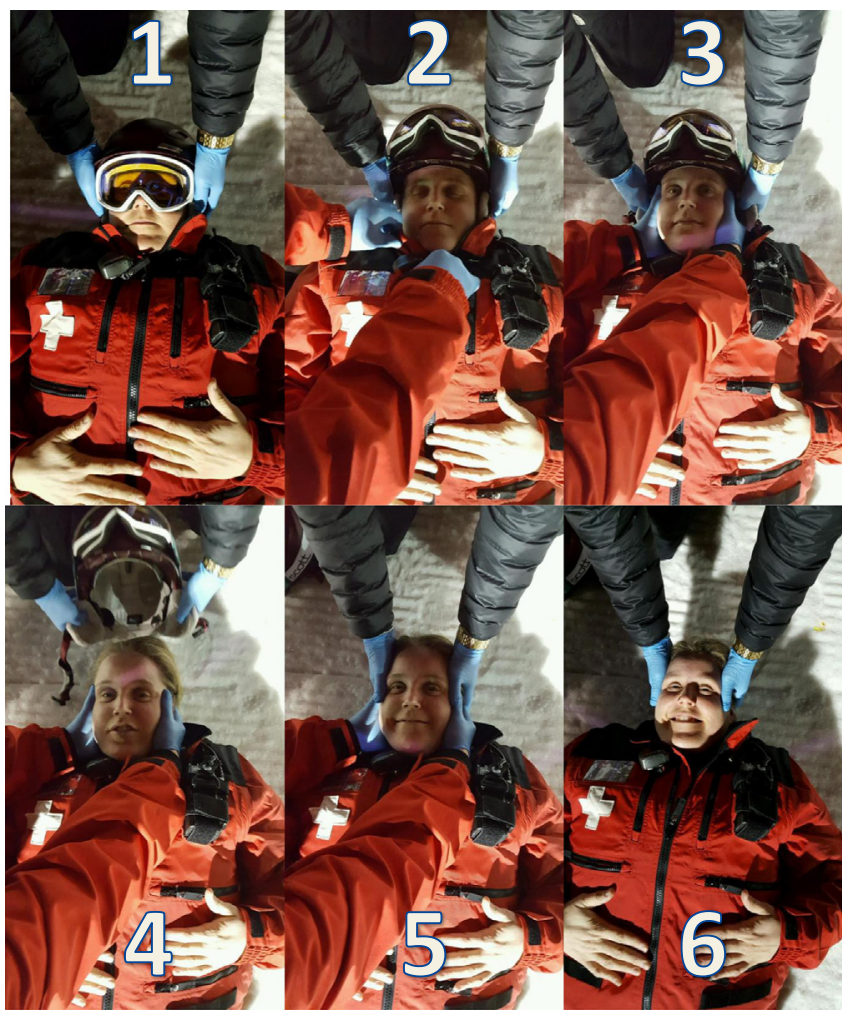

1) Rescuer 1 performs manual head/spine stabilization

2) Rescuer 2 removes goggles/straps

3) Rescuer 1 pulls outward, rescuer 2 places hands inside helmet and stabilizes head and spine from below

4) Rescuer 1 removes helmet

5) Rescuers 1 places hands on head from above

6) Rescuer 1 resumes manual head/spine stabilization

Figure 1. Two-person helmet removal technique.

each radiograph (Figure 2). The angles formed by the intersection of each of these 3 lines were then used to measure the occiput- $\mathrm{C} 2$ angle, $\mathrm{C} 2-\mathrm{C} 7$ angle, and
occiput-C7 angle. Positive angle values represent overall cervical extension whereas negative angle values represent overall cervical flexion.

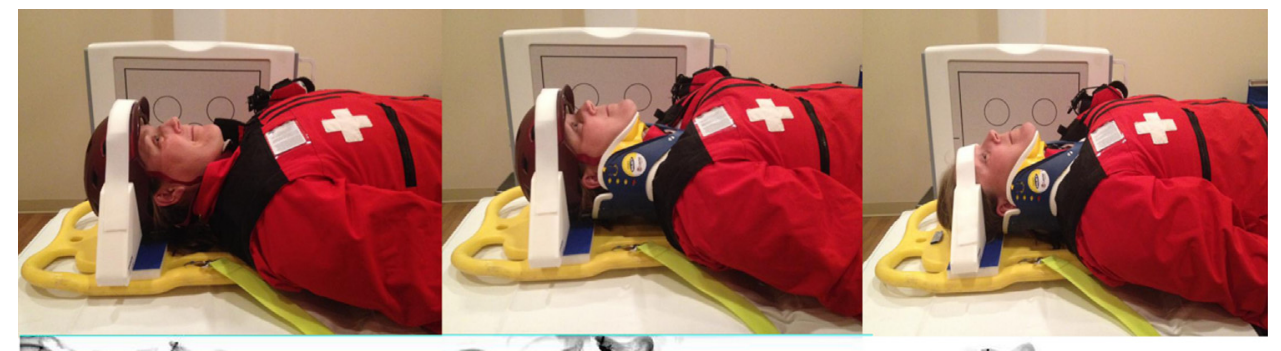

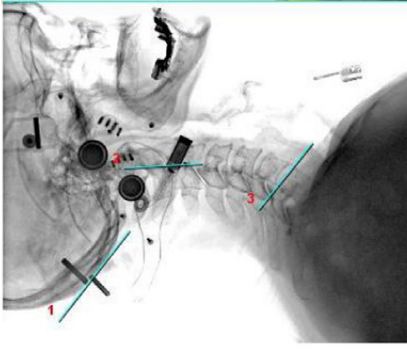

left: helmet on w/o collar

$1=$ posterior occiput line

Occiput-C2 angle $=<1,2$

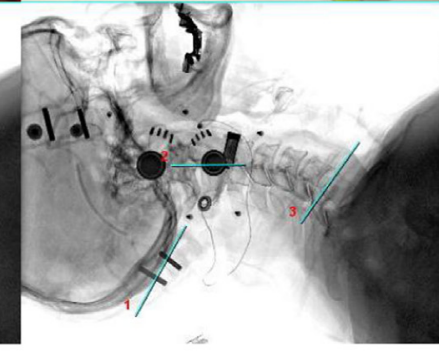

middle: helmet on, $w /$ collar

$2=$ posterior dens line

$\mathrm{C} 2-\mathrm{C} 7$ angle $=<2,3$

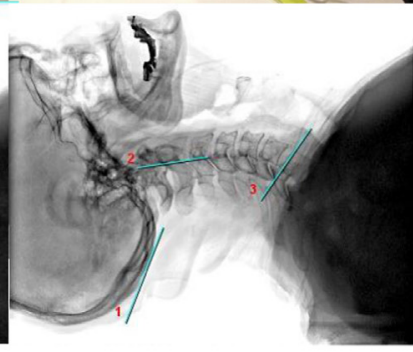

right: helmet off, $w /$ collar

$3=\mathrm{C} 7$ end-plate line

Occiput-C7 angle $=<1,3$

Figure 2. Spinal immobilization of helmeted skiers/snowboarders. 
Table 1. Mean values in degrees and (ranges) of overall and intersegmental cervical sagittal alignment

\begin{tabular}{llll}
\hline Mock rescue scenario & $O-C 2$ & $C 2-C 7$ & $O-C 7$ \\
\hline Helmet on, without collar (control) & $50(35-72)$ & $22(0-43)$ & $72(55-92)$ \\
Helmet on, with collar & $59(46-72)$ & $21(1-43)$ & $81(60-102)$ \\
Observed change in cervical extension & & 9 & $P=.001^{a}$ \\
& & $P=.52$ & $89(67-108)$ \\
Helmet off, with collar & & $30(5-51)$ & 17 \\
Observed change in cervical extension & Mean 10 min 38 sec & $P=.001^{a}$ & $P=.001^{a}$ \\
Time for helmet removal, c-collar, secure head & $\mathrm{SD} \pm 1: 38$ & Range 6:06-13:44 \\
\hline
\end{tabular}

$\mathrm{O}$, occiput; C, cervical.

$\kappa=0.85$ (excellent interrater reliability)

${ }^{a}$ Statistically significant.

Interrater reliability was assessed using Cohen's $\kappa$ coefficient and measurements for overall (occiputC7) and intersegmental sagittal alignment (occiput-C2, $\mathrm{C} 2-\mathrm{C} 7)$ were analyzed using analyses of variance and paired $t$ tests (Table 1). Statistical significance was set at $P<.05$.

\section{Results}

Compared with the control group, we observed a 9 degree increase in cervical extension when a collar was placed leaving the helmet on and a 17 degree increase when the helmet was removed and a collar placed, and these findings were statistically significant $(P=.001)$. We observed 9 degrees of increased extension at the occiput-C2 intersegment in both scenarios where a collar was placed $(P=.001)$. We only observed hyperextension at the $\mathrm{C} 2-\mathrm{C} 7$ intersegment in the scenario where the helmet was removed (8 degrees increased extension, $P=.001$ helmet off with collar vs 1 degree, $P=.52$ helmet on, with collar). Interrater reliability was excellent $(\kappa=0.85)$ between the 2 observers.
A subgroup analysis was also performed that identified anatomic differences in age and sex (Table 2). Women had increased cervical flexion compared with men overall and at all intersegments. Individuals aged $>50$ had increased cervical extension compared with those younger than 50 (Table 3). Age and sex did not, however, affect the changes observed in alignment when comparing control group with the helmet on, with ccollar and helmet off, with c-collar group.

In the timed portion of the scenario, candidate patrollers required 10 minutes, 38 seconds \pm 1:48 (8:20-13:38) (mean \pm SD with range) to remove the helmet, apply a cervical collar, and secure the head to the spinal backboard.

\section{Discussion}

In our study, both the application of a cervical collar as well as the removal of the ski helmet led to small but statistically significant changes in the static alignment of the cervical spine in the sagittal plane. When comparing the results with those of Swenson et al, the results are

Table 2. Gender subgroup analysis

\begin{tabular}{lllllll}
\hline Mock rescue scenario & Men O-C2 & Women $O-C 2$ & Men C2-C7 & Women C2-C7 & Men O-C7 & Women O-C7 \\
\hline \multirow{2}{*}{ Helmet on, without collar (control) } & 48 & 54 & 28 & 11 & 76 & 65 \\
Helmet on, with collar & 57 & 63 & 28 & 9 & 85 & 73 \\
Observed change in cervical extension & 9 & 10 & 0 & 2 & 10 & 8 \\
& $P=.001^{a}$ & $P=.001^{a}$ & $P=.854$ & $P=.477$ & $P=.001^{a}$ & $P=.041^{a}$ \\
Helmet off, with collar & 57 & 63 & 37 & 19 & 87 & 81 \\
Observed change in cervical extension & 8 & 9 & 8 & 8 & 17 & 16 \\
& $P=.000^{a}$ & $P=.003^{a}$ & $P=.001^{a}$ & $P=.002^{a}$ & $P=.001^{a}$ & $P=.001^{a}$ \\
\hline
\end{tabular}

O, occiput; C, cervical.

Measurements are in degrees.

${ }^{a}$ Statistically significant. 
Table 3. Age subgroup analysis

\begin{tabular}{|c|c|c|c|c|c|c|}
\hline Mock rescue scenario & $<50 \mathrm{O}-\mathrm{C2}$ & $>50 \mathrm{O}-\mathrm{C} 2$ & $<50 C 2-C 7$ & $>50 C 2-C 7$ & $<50 \mathrm{O}-\mathrm{C7}$ & $>50 \mathrm{O}-\mathrm{C7}$ \\
\hline Helmet on, w/o collar (control) & 54 & 46 & 16 & 29 & 69 & 75 \\
\hline Helmet on, w/ collar & 62 & 56 & 14 & 30 & 76 & 86 \\
\hline \multirow[t]{2}{*}{ Observed change in cervical extension } & 8 & 10 & 2 & 0 & 7 & 11 \\
\hline & $P=0.001^{*}$ & $P=0.001^{*}$ & $P=0.440$ & $P=0.960$ & $P=0.020^{*}$ & $P=0.001^{*}$ \\
\hline Helmet off, w/ collar & 61 & 56 & 24 & 38 & 84 & 94 \\
\hline \multirow{2}{*}{ Observed change in cervical extension } & 7 & 10 & 8 & 8 & 15 & 19 \\
\hline & $P=0.001^{*}$ & $P=0.001^{*}$ & $P=0.002^{*}$ & $P=0.001^{*}$ & $P=0.001^{*}$ & $P=0.001^{*}$ \\
\hline
\end{tabular}

Measurements are in Degrees

* Statistically Significant

remarkably similar. In their study, football players with shoulder pads and helmets were observed before and after helmet removal, and the helmet removal resulted in a 7.5 degree increase (25.3 with shoulder pads and helmets $[\mathrm{SH} / \mathrm{H}]$ vs 32.8 with shoulder pads $[\mathrm{SH}])$ in cervical extension from $\mathrm{C} 2-\mathrm{C} 7$. We observed an 8 degree increase in C2-C7 extension after helmet removal in our study ( 22 helmet on, without collar vs 30 helmet off, with collar). It suggests these findings may be applied to other helmeted athletes and supports the recommendation not to remove the helmet unless otherwise indicated.

The National Athletic Trainers Association has recently revised their protocol for the appropriate timing of helmet and equipment removal. ${ }^{20}$ Currently, they recommend an "all or none" approach to equipment removal. In their opinion, it is appropriate for team trainers and medical staff to remove the helmet, shoulder pads, and other equipment before transport only if such routines have been practiced and there are appropriate personnel to assist. Otherwise, the helmet and pads should be left in place to avoid unnecessary manipulation and malalignment.

For ski patrollers, equipment removal may depend on multiple factors involving the patient, rescuers, available equipment, and environmental factors. Keeping a helmet on the injured athlete can provide protection, warmth, and serve as a point of fixation for straps during a rescue. Equipment removal may not be practical or safe to perform at the rescue site, and it may be preferable to delay until the patient is in a warm, safe environment such as a patrol clinic, ambulance, or hospital. Absolute indications for helmet removal include a severely damaged or improperly fitting helmet that does not provide stability, restricted airway access, or bleeding/cerebrospinal fluid beneath the helmet that requires further evaluation (see Table 4 for indications and contraindications for helmet removal).

Applying a c-collar may also be unnecessary and potentially harmful. In our study, adding c-collar increased extension at occiput-C2 with relatively little effect on $\mathrm{C} 2-\mathrm{C} 7$ alignment. In helmeted athletes, significant variation in shape and styles can make it difficult to fit and secure a c-collar, potentially leading to undue manipulation of the spine. C-collars can also result in pressure sores and restricted access to the neck area, and prolonged use should generally be avoided if possible. In most rescue situations, the addition of a semifirm neck roll, such as a rolled towel or jacket placed securely under/adjacent to the cervical spine provides adequate support/stability without negatively affecting alignment. The primary role of the c-collar is to provide added stability to the spine. Thus, patrollers should only use a c-collar if they judge that adequate stability cannot be achieved without the collar (see Table 5 for pros and cons of applying a c-collar).

Table 4. Indications and contraindications for helmet removal

Absolute indications

Unable to establish airway

Open head wound or bleeding under helmet

Severely damaged/unstable helmet

Relative indications

Unorthodox helmet shape/size or attachment (camera)

Patient comfort (if alert)

Relative contraindications

C-spine injury with partial neurologic deficit (less manipulation)

Environmental factors

Cold temperature

High wind

Avalanche or rock fall danger

High angle, remote, or flight evacuation rescue

General considerations

Does rescuer have adequate experience and assistance to safely remove helmet?

Will removal delay rescue?

Will removal add benefit that outweighs risk of manipulation to spine? 
Table 5. Pros and cons of applying a cervical collar

Pros
Provides rigid (or semi rigid) stabilization
Cons
${ }^{a}$ Requires manipulation of spine and time to apply
If fixed or rigid spinal deformity or spasm, c-collar should
not be used
Need experience for efficient and proper fit
May not fit well with certain helmet styles
Pressure sores
Restricts access to neck area
Not always available

\footnotetext{
${ }^{a}$ Study found up to 10 minutes needed to perform helmet removal and c-collar application and average change in sagittal plane alignment of approximately 10 degrees with helmet removal and 20 degrees with helmet removal and c-collar application.
}

An additional finding in our study was the significant variation in "normal" anatomic alignment among healthy subjects. Our study revealed that both women and younger individuals had greater cervical flexion than men and those age $>50$. Despite these differences, we observed the same changes in alignment with both the addition of the c-collar and with removal of the helmet in all subgroups. We could not find any studies that evaluated the normal range or ideal spinal alignment in a c-collar and it is possible that our study is the first to show such a wide variation in normal C-spine alignment.
There are several limitations in this study. 1) Our study was performed in a classroom setting and our control group (helmet on, without c-collar) was assumed to be a reference to observe changes in alignment during a rescue. In reality, rescues occur in a multitude of locations and conditions, and patients would likely have significant variation in the position of the spine when found and/or could be moving or even combative during a rescue that would result in significantly more manipulation/movement of the spine than observed in our study. 2) Our study involved healthy volunteers, not patients with head/neck injuries, which would inherently affect alignment/stability. 3) Rescuers in our mock scenario were candidate patrollers completing the OEC course. Experienced patrollers would be expected to complete these tasks more efficiently and with less manipulation of the spine. 4) Our study accounts for static x-ray alignment only and does not measure the actual amount of motion that occurs during helmet removal and c-collar application. Such a study would provide useful information but would be technically difficult to perform.

In reality, there are a number of factors, sometimes unpredictable, that rescuers must use when determining how to safely and appropriately stabilize and transport injured patients with suspected head/neck injuries. ${ }^{24}$ Quinn et al recently authored the Wilderness Medical Society Practice Guidelines for Spine Immobilization in the Austere Environment. ${ }^{21}$ Our study findings are

Table 6. Spirit Mountain protocol for spinal injuries

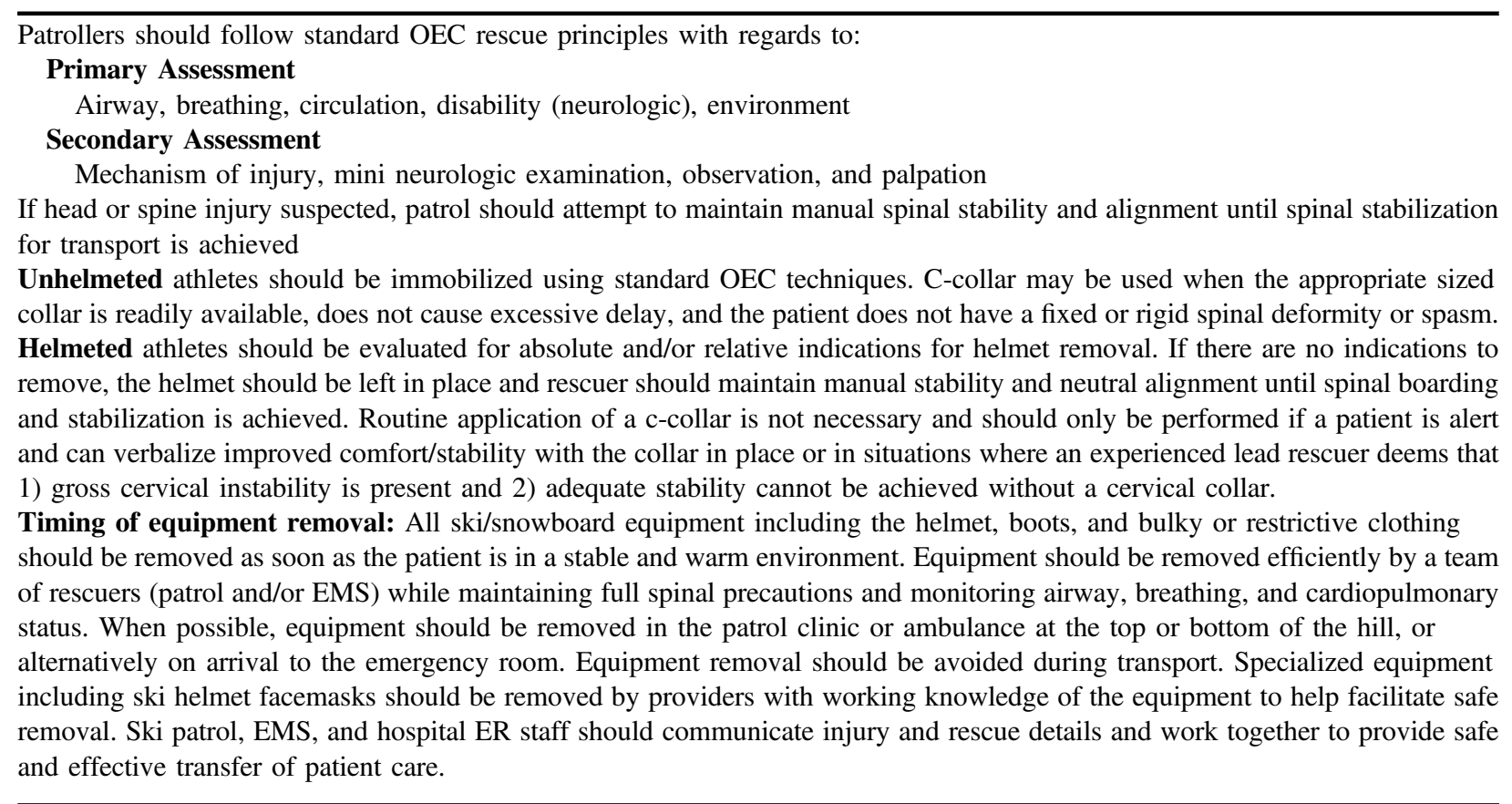


consistent with many of the clinical recommendations summarized by the Wilderness Medical Society. In particular, there is weak evidence to suggest that ccollars have any protective benefit for the injured spine and the authors' conclusions were similar to our own, urging rescuers to be very selective before placing a ccollar. The Wilderness Medical Society guidelines did not, however, address the specific issue of helmets and whether to remove them or leave them in place.

Another consideration is that not all ski patrollers utilize the same protocols. Although the National Ski Patrol primarily follows the OEC guidelines, there are a large number of professional ski patrollers that may have trained as paramedics, emergency medical technicians, or have other medical backgrounds such as nurses or physicians. Indeed, many patrols follow local protocols and although all patrols are recommended to have a medical advisor to review and update these protocols, there remains great variation in actual practice.

The future direction of spinal immobilization and transportation will most likely look at the replacement of long spinal boards and the implementation of vacuum mattress devices. ${ }^{22}$ These devices are malleable rubber full body length mats that are filled with numerous small beads that can be contoured snugly to the body, and when the air is sucked out (deflated) with a pump, the mattress becomes firm and can act as a full body splint. These devices have the advantage of being custom fit to each patient and avoid problems with pressure sores and other complications associated with spinal boards. These devices are widely used among European countries and in parts of the United States. We are currently investigating their use at our institution.

\section{Conclusions}

Our study strongly supports the practice of not removing the helmet of an injured skier/snowboarder with a suspected spine injury unless there are superseding factors. Additionally, our findings support the practice of avoiding routine use of c-collars unless a high index of suspicion exists for an unstable spinal injury. Our study provides scientific data that supports current 5th edition, Outdoor Emergency Care principals of ski patrol teaching. At our institution, we have implemented a protocol (Table 6) based on best available evidence that we believe may apply to a wide range of wilderness and environmental rescue situations.

Acknowledgments: Jim Gearns, OEC course instructor and Kristi Wilke, head of ski patrol at Spirit Mountain, Duluth, MN; Melissa Walls, PhD, Biostatistician, Associate Professor, Department of Biobehavioral Health and Population Sciences, University of Minnesota-
Duluth; Jaime Lahti, Catherine Nelson, and Kristyn Slattery, radiology technicians at St Luke's Orthopedics and Sports Medicine.

Author Contributions: Dr. David Rust is a sports medicine fellowship trained orthopedic surgeon and medical advisor to Spirit Mountain Ski Patrol and is responsible for the study design, IRB application, and study testing in coordination with the OEC course directors and is the article's lead author. Jared Murray is a medical student at the University of MinnesotaDuluth campus and assisted with volunteer consent, study testing, data/ statistical analysis, and with the review/editing of the manuscript.

Financial/Material Support: None.

Disclosure Statement: None.

\section{References}

1. SnowSports Industries America, Snow Sports Participation Report 2012. Available at: http://www.snowsports.org/. Accessed January 2017.

2. National Ski Areas Association, NSAA Fact Sheet [Internet] 2012. Available at: https://www.nsaa.org/media/68045/ NSAA-Facts-About-Skiing-Snowboarding-Safety-10-1-12. pdf. Accessed January 2017.

3. Rust D, Gilmore CJ, Treme G. Injury patterns at a large Western United States ski resort with and without snowboarders: the Taos experience. Am J Sports Med. 2013;41: 652-656.

4. National Spinal Cord Statistics Center, University of Alabama Birmingham Physical Medicine and Rehabilitation Department. Recent trends in causes of spinal cord injury. 2015. Available at: https://www.nscisc.uab.edu/ PublicDocuments/fact_sheets/Recent $\% 20$ trends $\% 20$ in $\%$ 20causes\%20of\%20SCI.pdf. Accessed January 2017.

5. Donelan S. Prehospital Medical Care and the National Ski Patrol. Wilderness Environ Med. 2012;23:175-176.

6. Constance B, Auerbach P, Johe D. Prehospital medical care and the National Ski Patrol: how does outdoor emergency care compare to traditional EMS training? Wilderness Environ Med. 2012;23:177-189.

7. McNamara E, Johe D, Endly D. Outdoor Emergency Care. 5th ed. London: Pearson; 2012.

8. Cusimano MD, Kwok J. The effectiveness of helmet wear in skiers and snowboarders: a systematic review. $\mathrm{Br} J$ Sports Med. 2010;44:781-786.

9. Haider AH, Saleem T, Bilaniuk JW. An evidence-based review: efficacy of safety helmets in the reduction of head injuries in recreational skiers and snowboarders. J Trauma Acute Care Surg. 2012;73:1340-1347.

10. Sulheim S, Holme I, Ekeland A. Helmet use and risk of head injuries in alpine skiers and snowboarders. JAMA. 2006;295:919-924.

11. Hagel B, Pless IB, Goulet C. The effect of helmet use on injury severity and crash circumstances in skiers and snowboarders. Acid Anal Prev. 2005;37:103-108.

12. Ruedl G, Burtscher M, Wolf M. Are self-reported risktaking behavior and helmet use associated with injury causes among skiers and snowboarders? Scand J Med Sci Sports. 2015;25:125-130.

13. Russell K, Chrisie J, Hagel B. The effect of helmets on the risk of head and neck injuries among skiers and snowboarders: a meta-analysis. CMAJ. 2010;182:333-340. 
14. Nova Scotia Legislature. Snow Sport Helmet Act. 2011, c. 47, s. 1. Nova Scotia: Authority of the Speaker of the House of Assembly Halifax; 2011.

15. Swenson TM, Lauerman WC, Blanc RO. Cervical spine alignment of immobilized football player. Radiographic analysis before and after helmet removal. Am J Sports Med. 1997;25:226-230.

16. Treme G, Diduch DR, Hart J. Cervical spine alignment in the youth football athlete. Recommendations for emergency transportation. Am J Sports Med. 2008;36: 1582-1586.

17. LaPrade RF, Schnetzler KA, Broxterman RJ. Cervical spine alignment in the immobilized ice hockey player. A computed tomographic analysis of the effects of helmet removal. Am J Sports Med. 2000;28:800-803.

18. Higgins M, Tierney RT, Driban JB. Lacrosse equipment and cervical spine space during immobilization: preliminary analysis. J Athl Train. 2010;45:39-43.

19. Radiological Society of North America, Inc, Radiation dose in $x$-ray and CT exams. 2014. Available at: https:// www.radiologyinfo.org/en/info.cfm?pg=safety-xray. Accessed January 2017.
20. National Athletic Trainers Association, Appropriate prehospital management of the spine-injured athlete. Updated as of August 5. Consensus Statement. 2015. Available at: https://www.nata.org/sites/default/files/executive-summaryspine-injury-updated.pdf. Accessed January 2017.

21. Quinn R, Williams J, Bennett B, Stiller G, Islas A, McCord S. Wilderness Medical Society practice guidelines for spine immobilization in the austere environment. Wilderness Environ Med. 2013;24:241-252.

22. Ellerton J, Tomazin I, Brugger H, Paal P. Immobilization and splinting in mountain rescue. Official Recommendations of the International Commission for Mountain Emergency Medicine, ICAR MEDCOM, Intended for Mountain Rescue First Responders, Physicians, and Rescue Organizations. High Alt Med Biol. 2009;10:337-342.

23. Fenerty L, Heatley J, Young J. Achieving all-age helmet use compliance for snow sports: a strategic use of education, legislation and enforcement. Inj Prev. 2016;22:176-180.

24. Johe D. Who should be put on a spine board with a cervical collar? In: Outdoor Emergency Care Refresher Workbook 2015 OEC Cycle B. Lakewood, CO: National Ski Patrol System Inc; 2015. 